\title{
Phase diagram of symmetric binary mixtures at equimolar and non-equimolar concentrations: a systematic investigation
}

\author{
D. Pini \\ Istituto Nazionale di Fisica della Materia and Dipartimento di Fisica, \\ Università di Milano, Via Celoria 16, 20133 Milano, Italy \\ M. Tau \\ Istituto Nazionale di Fisica della Materia and Dipartimento di Fisica, \\ Università di Parma, Parco Area delle Scienze 7/A, 43100 Parma, Italy

\begin{abstract}
A. Parola
Istituto Nazionale di Fisica della Materia and Dipartimento di Scienze Fisiche, Università dell'Insubria, Via Valleggio 11, 22100 Como, Italy
\end{abstract} \\ L. Reatto \\ Istituto Nazionale di Fisica della Materia and Dipartimento di Fisica, \\ Università di Milano, Via Celoria 16, 20133 Milano, Italy
}

\begin{abstract}
We consider symmetric binary mixtures consisting of spherical particles with equal diameters interacting via a hard-core plus attractive tail potential with strengths $\epsilon_{i j}, i, j=1,2$, such that $\epsilon_{11}=\epsilon_{22}>\epsilon_{12}$. The phase diagram of the system at all densities and concentrations is investigated as a function of the unlike-to-like interaction ratio $\delta=\epsilon_{12} / \epsilon_{11}$ by means of the hierarchical reference theory (HRT). The results are related to those of previous investigations performed at equimolar concentration, as well as to the topology of the mean-field critical lines. As $\delta$ is increased in the interval $0<\delta<1$, we find first a regime where the phase diagram at equal species concentration displays a tricritical point, then one where both a tricritical and a liquid-vapor critical point are present. We did not find any clear evidence of the critical endpoint topology predicted by mean-field theory as $\delta$ approaches 1 , at least up to $\delta=0.8$, which is the largest value of $\delta$ investigated here. Particular attention was paid to the description of the critical-plus-tricritical point regime in the whole density-concentration plane. In this situation, the phase diagram shows, in a certain temperature interval, a coexistence region that encloses an island of homogeneous, one-phase fluid.
\end{abstract}




\section{INTRODUCTION}

A major difference between the phase behavior of one-component fluids and binary mixtures is that, even if one considers just simple systems with a spherically symmetric, Lennard-Jones (LJ) like interaction profile, the qualitative features of the phase diagram of mixtures depend very sensitively on the parameters of the microscopic potential. If the interaction between a particle of species $i$ and a particle of species $j$ is modeled as the sum of a hard-core repulsion and a longer-ranged attractive tail, the relevant parameters are the hard-sphere diameters $\sigma_{i j}$ and the strengths $\epsilon_{i j}$ of the attractive contributions, $i, j=1,2$. This parameter space is drastically reduced by focusing on a particular class of systems, generally referred to as symmetric mixtures, such that $\sigma_{11}=\sigma_{22}=\sigma_{12}=\sigma, \epsilon_{11}=\epsilon_{22}=\epsilon$. Since the quantities $\sigma$ and $\epsilon$ can be included into the definition of the temperature $T$ and number densities $\rho_{i}$ by introducing standard reduced units $\rho_{i}^{*}=\rho_{i} \sigma^{3}, T^{*}=k_{\mathrm{B}} T / \epsilon, k_{\mathrm{B}}$ being the Boltzmann constant, it follows that the only parameter affecting the phase diagram is the ratio of the interaction strengths between unlike and like species $\delta=\epsilon_{12} / \epsilon$.

Clearly, symmetric mixtures appear quite artificial when considered as a model of real binary fluids. In fact, some of the features of their phase behavior hinge on the invariance with respect to the exchange of the two species, and are not found in the phase diagram of real mixtures. As has already been observed [1], symmetric mixtures are better seen as a model for a one-component fluid, whose particles have been endowed with a two-state, spin-like variable in addition to their translational degrees of freedom, so that their mutual interaction depends both on their relative position and on their "internal" state, namely whether the interacting particles belong to the same species or not. As such, this model

mixture is closely related to other models of dipolar [2,3] and magnetic [4, [, 6, 6] fluids, especially Ising-spin fluids [8,9,10]. In these systems, the phase behavior results from the interplay between the liquid-vapor phase separation and the additional transition, e.g. paraferromagnetic, associated to the spin-like degrees of freedom. In symmetric mixtures, the latter corresponds to the mixing-demixing transition.

Because of the relative simplicity of this model compared to a generic binary mixture and of the possibility of generating the whole spectrum of phase diagrams by acting on just one parameter, symmetric mixtures have been widely studied both by mean-field theory [1] and by numerical simulations [1, 11, 12, 13, 14, 15, 16]. A situation which has been given special attention is that of equal species concentration $x=\rho_{2} /\left(\rho_{1}+\rho_{2}\right)=1 / 2$. In this case, accurate numerical simulations [1] have qualitatively confirmed the mean-field scenario for the phase diagram as the parameter $\delta$ is varied in the interval $0<\delta<1$. This will be considered in detail in Secs. [II], IV. Here we just recall that the mean-field phase diagram at equal species concentration presents both a first-order coexistence boundary which separates a low-density fluid from a high-density one, and a line (the so-called $\lambda$-line) of mixingdemixing critical points. Beyond the $\lambda$-line, the fluid actually consists of two demixed fluids in equal amounts, one at a certain concentration $\bar{x}$, and the other at concentration $1-\bar{x}$, so that the overall concentration of the two species remains the same. For large enough $\delta$ $\left(\delta_{1}<\delta<1\right.$ with $\delta_{1}=0.708$ according to the mean-field result [1]) the coexistence curve ends into a liquid-vapor critical point, while the $\lambda$-line intersects the coexistence curve at a point of first-order transition, thereby terminating into a critical endpoint. At small $\delta$ $\left(0<\delta<\delta_{2}\right.$ with $\delta_{2}=0.605$ in mean field [1] $)$, on the other hand, the point at which 
the coexistence curve meets the $\lambda$-line coincides with its critical point. The latter is then referred to as a tricritical point, since on approaching this point from low temperatures, one observes the simultaneous coalescence of three phases, namely the low-density vapor and the two demixed high-density fluids. Finally, in a narrow interval of $\delta$ values $\delta_{2}<\delta<\delta_{1}$ intermediate between those corresponding to the two topologies described above, one has the occurrence of both a liquid-vapor critical point and a tricritical point. Despite the qualitative agreement between the mean-field scenario and the simulation results, there are still several points that deserve further investigation. First, mean-field theory and simulations show considerable quantitative discrepancies, which concern both the position of the critical loci and the values of $\delta$ at which the changes in the topology of the phase diagram occur. This is in itself not surprising, as mean-field theory cannot be expected to be quantitatively very accurate. Therefore, one would like to go beyond it by means of a theoretical treatment which includes fluctuations in the order parameter of the transition, be it of the liquid-vapor or mixing-demixing kind. Two relevant issues in this respect are whether the mean-field scenario is qualitatively recovered even after fluctuations have been taken into account, and which is the extent of the quantitative changes involved. Moreover, the case of equal species concentration corresponds just to a certain plane, albeit undoubtedly of special interest, of the space of thermodynamic states. Simulation studies of the phase diagram have indeed been performed also at fixed density $\rho$ and variable concentration $x$ [14, 16], but mapping the phase diagram in the whole thermodynamic space for different values of the parameter $\delta$ would require an exhorbitant number of simulation runs, and therefore hardly appears as a viable strategy in view of the computer time required. It is then tempting to resort to theory in order to explore the phase diagram at general density and concentration and find out how it changes by changing $\delta$, so as to see what the phase diagrams corresponding to the three regimes outlined above look like, as one moves away from the $x=1 / 2$ plane.

We aim to address these topics in the present work by means of the hierarchical reference theory (HRT) of binary fluids. This theory has already been successfully applied to the description of the critical behavior [17], crossover phenomena [17,18], and phase diagram [19] of simple fluid mixtures. Few results for the phase behavior of symmetric mixtures have also been reported [19,20], but a systematic investigation of this system by HRT has not been undertaken yet. In our opinion, HRT is especially well suited for such a study, with particular regard for the tasks stated above. In fact, the aim of this approach is determining how the Helmholtz free energy of the mixture is affected by the introduction of density and concentration fluctuations. This is achieved via a renormalization-group (RG) like procedure, where the long-wavelength Fourier components of the microscopic interaction are gradually introduced into the hamiltonian of the mixture. Any intermediate stage of this process, such that only Fourier components with wavevectors exceeding a certain cutoff $Q$ have been taken into account, physically corresponds to suppressing fluctuations on a lengthscale $L>1 / Q$. Long-range fluctuations are recovered in the limit $Q \rightarrow 0$, when the free energy of the fully interacting system is obtained, while the mean-field free energy enters as the initial condition at $Q=\infty$. The main advantage of HRT over other liquid-state theories is that it embodies several features of the RG description of critical phenomena in a treatment based on the microscopic hamiltonian of the fluid. These include scaling, nontrivial critical exponents, and the correspondence between universality classes and different fixed points of the RG flow [17]. A fact of particular relevance for the investigation pursued 
here is that the inclusion of long-range fluctuations has the effect of preserving the correct convexity of the free energy in the whole thermodynamic space. Whenever phase coexistence occurs, one does not find any domain of instability as in the mean-field approximation, and the conditions of thermodynamic equilibrium between the phases at coexistence are enforced by the theory itself. At each given temperature, the coexistence region is then immediately recovered as the locus in the density-concentration plane where the chemical potential of each component is constant along the lines of fixed pressure, with no need of imposing this condition a posteriori by a Maxwell construction. For binary mixtures, the latter proves to be quite cumbersome already at the mean-field level, and is much more so for more sophisticated integral-equation theories, in which the occurrence of phase separation generally entails the presence of some forbidden domain, where the theory cannot be solved at all. Therefore, the ability of straightforwardly mapping the phase diagram is a valuable asset of HRT. This is especially true in the present case where the topology of the phase diagram is very sensitive to changes in $\delta$, while at the same time, as will be seen in the following, the features that allow one to discriminate between different topologies are often detectable only in a narrow window of the thermodynamic space. Because of the lack of a solution defined for every $(T, \rho, x)$ state, pinpointing all these features by conventional integral-equation theories would undoubtedly prove extremely difficult, perhaps even impossible.

In this work we have considered symmetric mixtures of additive hard spheres interacting via an attractive Yukawa tail potential $w_{i j}(r)=-\sigma \epsilon_{i j} e^{-z(r / \sigma-1)} / r$, where $r$ is the interparticle distance and $z$ is the inverse range of the interaction, which has been fixed to the value $z=1.8$ for both like and unlike species. This hard-core Yukawa (HCY) form has been preferred to the square-well potential used in Ref. [1]. The latter lends itself well to simulation, but the very slowly decaying behavior of its Fourier transform makes it somewhat tedious to use in HRT. The HCY potential has already been adopted in a number of studies of symmetric mixtures based on the mean spherical approximation (MSA) [21], the optimized random phase approximation (ORPA) [22], and the self-consistent Ornstein-Zernike approximation (SCOZA) [23], all of which yielded for the phase diagram at equimolar concentration the same behavior found in mean-field theory. The scenario that comes out of our investigation by HRT agrees qualitatively with the mean-field one in predicting that, as $\delta$ is increased, the phase diagram at equimolar concentration exhibits first a tricritical point, and subsequentely both a tricritical and a liquid-vapor critical point. However, we did not find any clear evidence of the occurrence of the mixing-demixing critical endpoint given by mean-field theory as $\delta$ approaches 1 , at least up to $\delta=0.8$, above which further investigation is hindered by the finite resolution of the density grid used in our numerical calculation. Besides the scenario sketched above at equal species concentration, other interesting features of the phase diagram emerge as we move off the $x=1 / 2$ axis. For instance, the intermediate regime where both a critical and a tricritical point are found for $x=1 / 2$ is marked in the $\rho-x$ plane by a coexistence region which in a certain temperature interval contains a "hole" or "island" of homogeneous, mixed fluid. Double critical points and, for a certain value of $\delta$, tricritical points are also observed for unequal species concentration.

The paper is structured as follows: the HRT for a binary fluid is described in Sec. [1. Mean-field theory is recovered within this approach as a zeroth-order approximation, and the mean-field results for the critical lines of a symmetric mixture as the parameter $\delta$ is varied in the interval $0<\delta<1$ are shown in Sec. III. The HRT phase diagram for different 
values of $\delta$ is discussed and compared with the mean-field predictions in Sec. IV]. Finally, in Sec. $\mathrm{V}$ we summarize our findings and draw our conclusions.

\section{HRT EQUATIONS}

Here we briefly review the HRT approach for a binary fluid. A more detailed derivation can be found in previous works [17, 19,24].

We consider a model mixture consisting of particles of two species interacting via a twobody spherically symmetric potential $v_{i j}(r)$, where the indices $i, j$ label the particle species. The derivations presented in this Section do not hinge on the fact that one is dealing with a symmetric system such that $v_{11}(r)=v_{22}(r)$, so they are equally valid for non-symmetric systems. We assume that $v_{i j}(r)$ can be split as the sum of a singular contribution $v_{i j}^{\mathrm{R}}(r)$ which accounts for the short-range repulsion between the particles, and a longer-ranged, smooth attractive tail $w_{i j}(r)$ which may induce fluid-fluid phase separation. The properties of the mixture interacting via the repulsive potential $v_{i j}^{\mathrm{R}}(r)$ alone are considered as known, so that it acts as a "reference" or unperturbed system. For a hard-core plus tail potential, the natural choice for the reference system is just a binary mixture of additive hard spheres, which can be described by the Mansoori-Carnahan-Starling-Leland equation of state [25] and the corresponding Verlet-Weis parameterization for the two-body correlations [26,27]. We remark that here we will not be concerned with a possible demixing transition occurring in the hard-sphere mixture, since such a transition may come along only as a consequence of depletion interactions when the particles differ widely in size. In the present case of equisized particles, the reference system reduces to a one-component hard-sphere fluid, and all the fluid-fluid transitions displayed by the system are necessarily due to the attractive perturbation $w_{i j}(r)$. The HRT differs from the conventional liquid-state approaches in the way this perturbation is dealt with. In order to accurately describe the long-range fluctuations that are important in criticality and phase separation, the attractive part of the interaction is switched on gradually by introducing a $Q$-system with a modified interaction $v_{i j}^{Q}(r)=v_{i j}^{\mathrm{R}}(r)+w_{i j}^{Q}(r)$, where $w_{i j}^{Q}(r)$ is defined in such a way that its Fourier transform $\widetilde{w}_{i j}^{Q}(k)$ coincides with that of the original attractive potential $\widetilde{w}_{i j}(k)$ for $k>Q$, and vanishes for $k<Q$. Inspection of the diagrammatic series of the Helmholtz free energy of the mixture in terms of the perturbation $w_{i j}(r)$ and the correlation functions of the reference system shows that introducing such an infra-red cutoff in the interaction is physically equivalent to inhibiting fluctuations with characteristic lengths $L>1 / Q$. If $Q$ is made evolve from $Q=\infty$, the $Q$-systems evolve from the reference system by acquiring fluctuations of longer and longer wavelengths. The fully interacting system is recovered as the $Q \rightarrow 0$ limit of such a process. Only in this limit true long-range correlations are allowed to develop in the fluid. The equation for the corresponding evolution of the Helmholtz free energy $A_{Q}$ of the $Q$-systems can be determined exactly and is related to the attractive perturbation in momentum space $\Phi_{i j}(k)=-\beta \widetilde{w}_{i j}(k)$ where $\beta=1 /\left(k_{\mathrm{B}} T\right)$, and to the direct correlation function of the $Q$-system in momentum space $c_{i j}^{Q}(k)$. We recall that the direct correlation function is related to the structure factor of the fluid by the Ornstein-Zernike relation. If we denote by $c_{Q}(k)$ the $2 \times 2$ symmetric matrix with elements $c_{i j}^{Q}(k)$, for a binary mixture this relation reads 


$$
\left[c_{Q}^{-1}(k)\right]_{i j}=-\sqrt{\rho_{i} \rho_{j}} S_{i j}^{Q}(k),
$$

where $c_{Q}^{-1}(k)$ is the inverse matrix of $c_{Q}(k)$ and $S_{i j}^{Q}(k)$ is the partial structure factor of the $Q$-system. Here, unlike in the convention commonly adopted in liquid-state theory, $c_{i j}^{Q}(k)$ contains its ideal-gas contribution $-\delta_{i j} / \rho_{i}$. The evolution equation for the Helmholtz free energy is most easily formulated in terms of a modified free energy $\mathscr{A}_{Q}$ and direct correlation function $\mathscr{C}_{Q}(k)$ defined as:

$$
\begin{aligned}
\mathscr{A}_{Q} & =-\frac{\beta A_{Q}}{V}+\frac{1}{2} \sum_{i, j=1}^{2} \rho_{i} \rho_{j}\left[\Phi_{i j}(k=0)-\Phi_{i j}^{Q}(k=0)\right]-\frac{1}{2} \sum_{i=1}^{2} \rho_{i} \int \frac{d^{3} \mathbf{k}}{(2 \pi)^{3}}\left[\Phi_{i i}(k)-\Phi_{i i}^{Q}(k)\right] \\
\mathscr{C}_{i j}^{Q}(k) & =c_{i j}^{Q}(k)+\Phi_{i j}(k)-\Phi_{i j}^{Q}(k) .
\end{aligned}
$$

The HRT equation for $\mathscr{A}_{Q}$ is then

$$
\frac{\partial \mathscr{A}_{Q}}{\partial Q}=-\frac{Q^{2}}{4 \pi^{2}} \log \left\{\operatorname{det}\left[\mathbf{1}-\mathscr{C}_{Q}^{-1}(Q) \Phi(Q)\right]\right\},
$$

where again $\Phi(Q)$ and $\mathscr{C}_{Q}^{-1}(Q)$ are $2 \times 2$ symmetric matrices, the latter being the inverse of the matrix $\mathscr{C}_{Q}(Q)$. We note that for $Q \rightarrow 0$, i.e. at the end of the evolution process, $\Phi_{Q}(k)$ and $\Phi(k)$ coincide, so that in this limit the modified quantities $\mathscr{A}_{Q}, \mathscr{C}_{Q}$ yield respectively the true free energy and direct correlation function of the fully interacting system. For $Q=\infty$ instead one has $\Phi_{Q}(k) \equiv 0$, and $\mathscr{A}_{Q}$ and $\mathscr{C}_{Q}$ are nothing but the mean-field free energy and the random-phase approximation (RPA) direct correlation function in the presence of the full perturbing potential $\Phi(k)$. These play the role of the initial conditions of the evolution equation (बit), which then describes how the mean-field estimate for the free energy is affected by the inclusion of fluctuations. This equation is manifestly not closed, since the evolution of the free energy $\mathscr{A}_{Q}$ is related to the matrix of the direct correlation function $\mathscr{C}_{Q}(k)$, which is itself unknown. In fact, Eq. (4) is just the first equation of an infinite hierarchy for the direct correlation functions of increasing order: for instance, the evolution of $\mathscr{C}_{Q}(k)$ is related to the 3- and 4-body direct correlation functions in Fourier space [24]. A point of crucial importance in order to implement a viable HRT scheme consists then in supplementing Eq. (4) with some closure relation involving $\mathscr{C}_{Q}(k)$. Here, as well as in the previous applications of HRT, we have not resorted to the higher-order equations of the hierarchy. Instead, we have adopted for $\mathscr{C}_{Q}(k)$ an approximate form inspired by standard perturbative liquid-state theories:

$$
\mathscr{C}_{i j}^{Q}(k)=c_{i j}^{\mathrm{HS}}(k)+\nu_{i j}^{Q} \Phi_{i j}(k),
$$

where $c_{i j}^{\mathrm{HS}}(k)$ is the Fourier transform of the partial direct correlation function of the hardsphere reference system, which has been represented by the above-mentioned Verlet-Weis parameterization. The functional form of Eq. (5) for the direct correlation function is similar to that of the widely used RPA, which is recovered for $\nu_{i j}^{Q}=1$. In particular, both of them rely on the Ornstein-Zernike ansatz, i.e., the direct correlation function has always the same range as the potential, so that $\mathscr{C}_{i j}^{Q}(k)$ is always analytic in $k$, including at the critical points of the system, where the real direct correlation function is instead expected to be nonanalytic for $Q \rightarrow 0$. However, unlike in the RPA, the amplitude $\nu_{i j}^{Q}$ of the perturbation 
is regarded as an unknown quantity, to be determined in such a way that each $Q$-system satisfies the compressibility sum rule. In a one-component fluid, this relates the isothermal compressibility to the zero-wavevector value of the structure factor, or equivalently of the direct correlation function in momentum space. Such a rule is readily generalized to binary systems, and in terms of the modified quantities $\mathscr{A}_{Q}, \mathscr{C}_{Q}(k)$ it reads

$$
\mathscr{C}_{i j}^{Q}(k=0)=\frac{\partial^{2} \mathscr{A}_{Q}}{\partial \rho_{i} \partial \rho_{j}} \quad i, j=1,2 .
$$

By determining $\nu_{i j}^{Q}$ so that Eq. (河) is satisfied, one immediately finds from Eq. (5)

$$
\mathscr{C}_{i j}^{Q}(k)=c_{i j}^{\mathrm{HS}}(k)+\left[\frac{\partial^{2} \mathscr{A}_{Q}}{\partial \rho_{i} \partial \rho_{j}}-c_{i j}^{\mathrm{HS}}(k=0)\right] \varphi_{i j}(k),
$$

where we have set $\varphi_{i j}(k)=\Phi_{i j}(k) / \Phi_{i j}(k=0)$. Eq. (面) ensures that the Helmholtz free energy obtained by integration of Eq. (4) is consistent with the compressibility route to the thermodynamics given by Eq. (6). This thermodynamic consistency condition plays a key role in the implementation of HRT. In fact, by substituting $\mathscr{C}_{i j}^{Q}(k)$ as given by Eq. (7) into the HRT equation (4), a closed partial differential equation (PDE) for $\mathscr{A}_{Q}$ is obtained, which involves both the first partial derivative of $\mathscr{A}_{Q}$ with respect to $Q$, and its second partial derivatives with respect to the densities $\rho_{1}, \rho_{2}$. In order to integrate this equation numerically, we found it most convenient to cast it into a form where the partial derivatives of the unknown function appear only outside some "coefficients" that may depend both on the independent variables $\rho_{1}, \rho_{2}, Q$, and on the unknown function itself, but do not contain its derivatives. This allows us to take advantage of finite-difference schemes especially devised for equations of such a quasi-linear form, which combine robustness with a moderate computational cost [28]. Both of these are very important requirements in our case. In fact, in order to deal with the divergence of the compressibility at criticality and phase coexistence, one has to resort to a very stable algorithm, while on the other hand for a diffusive PDE in three independent variables like the one considered here, the dimension of the vectors generated by the discretization procedure becomes rapidly very large even for a relatively coarse density step $\Delta \rho$. Hence, we have to use a solution scheme which is not too demanding computationally to prevent computer time from increasing beyond control.

The method we adopted to re-write Eq. (田) supplemented by the closure relation (7) in quasi-linear form has already been illustrated in Ref. [19, to which we refer the reader for details. Here we recall that, instead of $\mathscr{A}_{Q}$, we use as unknown function the quantity

$$
U=\log \left\{\operatorname{det}\left[\mathbf{1}-\mathscr{C}_{Q}^{-1}(Q) \Phi(Q)\right]\right\}
$$

This is found to satisfy a quasi-linear PDE of the form

$$
e^{U} \frac{\partial U}{\partial Q}=K \frac{\partial^{2} U}{\partial \rho_{1}^{2}}+L \frac{\partial^{2} U}{\partial \rho_{1} \partial \rho_{2}}+M \frac{\partial^{2} U}{\partial \rho_{2}^{2}}+N
$$

whose "coefficients" $K, L, M, N$, which will not be reported here, depend on the variables $\rho_{1}, \rho_{2}, Q$ both explicitly and implicitly via a set of three auxiliary variables. These can be identified either with the eigenvalues $\lambda_{1}, \lambda_{2}$ of the symmetric matrix $\mathscr{C}_{Q}(Q)$ and the angle 
$\alpha$ of the rotation that casts $\mathscr{C}_{Q}(Q)$ into diagonal form, or with the corresponding quantities $\Lambda_{1}, \Lambda_{2}, \theta$ for the symmetric matrix of elements $\partial^{2} \mathscr{A}_{Q} / \partial \rho_{i} \partial \rho_{j}$. As discussed in [19], the use of one or the other set of auxilary variables is dictated by the different behavior of the interaction in the high- and low- $Q$ region. At high $Q$, where the Fourier transform of the attractive perturbation $\varphi_{i j}(Q)$ typically displays oscillations, thereby vanishing for certain $Q$ values, it is better to adopt as auxiliary quantities $\Lambda_{1}, \Lambda_{2}, \theta$. At small $Q$ instead, when most of the attractive interaction has been included into the system and phase coexistence may occur, the set $\lambda_{1}, \lambda_{2}, \alpha$ is the more appropriate choice. In both cases, the derivative of each of the auxilary variables with respect to $Q$ can be expressed in terms of the variables themselves and the partial derivatives of $U$ with respect to $\rho_{1}, \rho_{2}$. At any given $Q$, the resulting equations are used to update $\lambda_{1}, \lambda_{2}, \alpha$ (or $\Lambda_{1}, \Lambda_{2}, \theta$ ), while the PDE (9) is used to update $U$. Since the matrix $\mathscr{C}_{Q}(Q)$ that appears in Eq. (8) can be expressed either by $\lambda_{1}$, $\lambda_{2}, \alpha$ or, via Eq. (7), $\Lambda_{1}, \Lambda_{2}, \theta$, it follows that $U$ and the set of the three auxiliary variables are not mutually independent. The relation between $U$ and the auxiliary variables is used throughout the integration procedure as a check of the accuracy of the calculation.

In order to perform the numerical integration, we found it convenient to replace the independent variables $\rho_{1}, \rho_{2}$ with the related variables $\xi=\left(\rho_{1}+\rho_{2}\right) \sigma^{3}, x=\rho_{1} /\left(\rho_{1}+\rho_{2}\right)$. At any given $Q, U$ is then defined in the rectangular domain $0 \leq x \leq 1,0 \leq \xi \leq \xi_{\max }$, where the high-density boundary $\xi_{\max }$ was chosen equal to 1 . At the beginning of the evolution process, i.e. for suitably large $Q$, the matrix $\mathscr{C}_{Q}(k)$ can be identified with the RPA expression for the direct correlation function which, as said above, is obtained from Eq. (3) for $Q=\infty$. This gives the initial condition for $U$ via Eq. (8). The boundary conditions for $U$ needed for the integration of Eq. (9) are determined as follows: for $\xi=0$ the diagonal elements of the matrix $\mathscr{C}_{Q}(Q)$ diverge because of the ideal-gas contribution to the direct correlation function $-\delta_{i j} / \rho_{i}$, so that $U(\xi=0, x)$ vanishes identically. For $x=0$ and $x=1$ only one of the two species is present. The corresponding boundary conditions are then given by the solution of the HRT equation for a pure fluid, which can be integrated numerically by specializing the procedure sketched above to a one-component system. The high-density boundary condition for $\xi=\xi_{\max }$ is non-trivial because, unlike in pure fluids, we must allow the occurrence of phase transitions even at high density. As a consequence, we have to rule out the possibility of using for $U$ at $\xi=\xi_{\max }$ a simple approximation such as the RPA, as already done for the one-component case, because such a form would behave unphysically in the coexistence region. In general, we expect that at high density the compressibility of the fluid will be small, and concentration fluctuations will become more important than density ones. This corresponds to the transition becoming less liquid-vapor and more mixingdemixing in character. For the symmetric mixtures considered here, such an expectation holds rigorously: in fact, because of the special symmetry of the model, the high-density transition are of pure mixing-demixing type. As a consequence, we expect $U$ to be much more sensitive to a change in concentration than in density. Accordingly, for $\xi=\xi_{\max }$ the partial derivatives of $U$ along $\xi$ in Eq. (9) have been disregarded with respect to those along the conjugate direction $z=\left(\rho_{1}-\rho_{2}\right) \sigma^{3}$. By switching from the variables $\xi, z$ back to the variables $\xi, x$, we obtain a PDE in the variables $Q, x$ on the boundary $\xi=\xi_{\max }$, whose solution yields the high-density boundary condition of Eq. (9).

The numerical integration of Eq. (9) supplemented by the equations for the evolution of the auxilary variables was performed on a grid in the $(\xi, x)$ plane containing $150 \times 150$ 
mesh points. The integration with respect to the variable $Q$ was carried out by setting $Q=Q_{0} e^{-t}, t \geq 0$, where the initial value of $Q$ corresponding to $t=0$ was typically fixed at $Q_{0}=30 \sigma^{-1}$. The variable $t$ was discretized using a step $\Delta t=10^{-3}$ and the iteration in $t$ went on until convergence in the quantity $U$ was achieved outside the coexistence region. At low temperature this requirement can be satisfied for $Q<10^{-4} \sigma^{-1}$.

\section{MEAN-FIELD CRITICAL LINES}

The phase diagrams of binary mixtures are usually classified according to the topology of their critical lines [29,30]. We then begin the discussion of the phase behavior of the symmetric mixtures as a function of the interaction parameter $\delta$ by presenting the different shapes of the critical lines that are predicted by the mean-field approximation. We will then consider the HRT results for the phase diagram and elucidate the relationship between the critical loci and the behavior of the coexistence domains on changing the temperature. In doing so, we will also be in a position to compare the mean-field and HRT predictions.

As observed above, the mean-field (MF) approximation enters the HRT as the initial condition at $Q=\infty$, when no fluctuations have been introduced into the system:

$$
-\frac{\beta A_{\mathrm{MF}}}{V}=\mathscr{A}_{Q=\infty}=-\frac{\beta A_{\mathrm{HS}}}{V}+\frac{1}{2} \sum_{i, j=1}^{2} \rho_{i} \rho_{j} \Phi_{i j}(k=0)-\frac{1}{2} \sum_{i=1}^{2} \rho_{i} \int \frac{d^{3} \mathbf{k}}{(2 \pi)^{3}} \Phi_{i i}(k) .
$$

The equations for the MF critical loci are also obtained within HRT as the lowest-order approximation to the requirement that the RG flow generated by the evolution equation (四) drives the free energy of the mixture towards its fixed point. This gives the following equations [17]:

$$
\begin{aligned}
& \frac{\partial^{2}}{\partial \psi_{1}^{2}}\left(\frac{\beta A_{\mathrm{MF}}}{V}\right)=0, \\
& \frac{\partial^{3}}{\partial \psi_{1}^{3}}\left(\frac{\beta A_{\mathrm{MF}}}{V}\right)=0, \\
& \frac{\partial^{2}}{\partial \psi_{1} \partial \psi_{2}}\left(\frac{\beta A_{\mathrm{MF}}}{V}\right)=0,
\end{aligned}
$$

where $\psi_{1}, \psi_{2}$ are obtained from the original densities $\rho_{1}, \rho_{2}$ via an a priori unknown rotation such that Eq. (13) is satisfied. Eqs. (11), (12) are formally similar to the equations for the critical point of a pure fluid, except that here the density $\rho$ has been replaced by the variable $\psi_{1}$. We note that Eqs. (11), (13) amount to requiring that the hessian determinant of the free energy must vanish at the critical point, and that the vanishing eigenvalue corresponds to the eigenvector directed along $\psi_{1}$. This is the linear combination of the densities that gives the direction of strongest fluctuation, and identifies the order parameter of the transition. For the solutions of Eqs. (11)-(13) to yield actual critical points, a further constraint has to be imposed, i.e. the condition of thermodynamic stability ensuring that even a critical point is an equilibrium state of the system, and as such is stable against density and concentration fluctuations. The stability conditions at a critical point read [17] 


$$
\begin{aligned}
& \frac{\partial^{2}}{\partial \psi_{2}^{2}}\left(\frac{\beta A_{\mathrm{MF}}}{V}\right)>0, \\
& {\left[\frac{\partial^{3}}{\partial \psi_{1}^{2} \partial \psi_{2}}\left(\frac{\beta A_{\mathrm{MF}}}{V}\right)\right]^{2}-\frac{1}{3} \frac{\partial^{2}}{\partial \psi_{2}^{2}}\left(\frac{\beta A_{\mathrm{MF}}}{V}\right) \frac{\partial^{4}}{\partial \psi_{1}^{4}}\left(\frac{\beta A_{\mathrm{MF}}}{V}\right)<0 .}
\end{aligned}
$$

The three equations (11)-(13) with the conditions (14), (15) contain four unknowns, namely $\rho_{1}, \rho_{2}, T$, and the state-dependent angle $\gamma$ of the rotation that identifies the proper axes $\psi_{1}, \psi_{2}$. As $\gamma$ is varied, they will generate a set of lines in the thermodynamic space. We must observe that, when several critical lines are present, one should also check that a critical point does not fall into the coexistence region originating from another critical line. In mean-field theory, this circumstance can occur without violating the stability conditions (14), (15), when the point considered lies between the binodal and the spinodal surfaces of a neighboring transition. In such a situation, a solution of Eqs. (11)-(15) corresponds to a critical point which, while still locally stable, is however globally metastable with respect to first-order phase separation. In order to assess this possibility, one should then determine the mean-field binodal surfaces by a Maxwell construction. This has not been done here. However, we can discriminate between stable and metastable regimes, at least for equimolar concentrations, by comparing our results with those obtained in Ref. [1], where mean-field binodals were determined. We recall that at the mean-field level the phase behavior as a function of $\delta$ is independent of the profile of the attractive interaction $\Phi_{i j}$, since this enters in the approximation only via its spatial integral. A change in the form of $\Phi_{i j}$ is then taken into account by simply rescaling the temperature.

Let us now consider the critical lines of the symmetric mixtures we are interested in. Because of the attractive interaction between the particles, we expect that for suitable temperature, density, and concentration, the system will undergo a liquid-vapor transition. This is certainly true for states at low or high concentration, where one of the two species will play a minor role. On the other hand, since the interaction between unlike particles is weaker than that between like particles, the internal energy will tend to promote demixing between the two species. For high enough density, this increase in the absolute value of the internal energy may overcome the loss in entropy implied by the demixing, and a mixing-demixing transition may appear. Therefore, both liquid-vapor and mixing-demixing critical lines are expected. As the unlike-to-like interaction ratio $\delta$ is lowered, the mixing-demixing transition becomes more favored, and the corresponding critical line will move to lower density. The projections of the mean-field critical lines on the density-concentration plane for a relatively

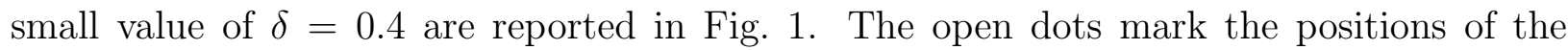
minima of the critical temperature, while the arrows drawn along the critical lines give a graphic representation of the relative weight of density and concentration fluctuations, thereby showing the direction of the order parameter $\psi_{1}$ determined by Eqs. (11)-(13). Specifically, the angle $\phi$ between the arrows and the density axis gives the fluctuation of the order parameter corresponding to a given fluctuation of the total density $\rho$ and concentration $x$ as $\delta \psi_{1}=\delta \rho \cos \phi+\rho \delta x \sin \phi$. Arrows parallel to the $\rho$ - and $x$-axis then indicate pure liquidvapor and mixing-demixing transitions, respectively. Fig. 1 shows a mixing-demixing critical line at equal species concentration $x=1 / 2$. On the low-density side, this intersects another critical line that connects the critical points of the pure species. As one moves from the pure species to the equimolar mixture, the direction of the order parameter changes continuously 
from pure liquid-vapor to pure mixing-demixing. The critical temperature, not shown in the figure (see the lower panel of Fig. 9 for the similar case $\delta=0.5$ ), initially decreases until it reaches a minimum at two points symmetric with respect to $x=1 / 2$, after which it starts increasing and keeps on doing so along the mixing-demixing line as the density is increased. This behavior of the critical temperature is related to the change in the order parameter of the transition. Close to $x=0$ or $x=1$, where phase separation is essentially of liquid-vapor type, increasing the amount of the dilute component increases the weight of the interaction between unlike species in the internal energy. Since here we have $\delta<1$, this leads to a decrease of the overall attractive contribution to the internal energy, resulting in a lower critical temperature of the liquid-vapor transition. On the other hand, the same argument implies that for a transition which is mainly mixing-demixing in character, approaching equimolar concentration increases the gain in internal energy entailed by the demixing, so that the critical temperature increases as one moves towards $x=1 / 2$. Once the mixingdemixing critical line has been reached, an increase of the density at constant concentration similarly favors the energetic contribution to the free energy and leads to an increase of the critical temperature.

As $\delta$ is increased, the mixing-demixing line, as noted above, moves to higher density, and so does that portion of the line originating from the pure species where the transition is predominantly of demixing type. At about $\delta=0.46$, a new feature appears in the critical lines, namely a crescent-shaped line at low density and concentration spanning an interval centered at $x=1 / 2$, where the transition is essentially liquid-vapor. This is shown in Fig. 2 for $\delta=0.65$. The occurrence of such a critical line can be euristically explained as follows: if $\delta$ were equal to 1 , the mixture would reduce to a one-component hard-sphere fluid with attractive tail interaction. If $\delta$ is not too small, a nearly equimolar mixture may still behave like a sort of "effective" one-component fluid displaying a liquid-vapor transition. In order for this to happen, however, the density has to be low enough, so that the smaller gain in internal energy resulting from choosing a liquid-vapor phase separation at intermediate concentration instead of a mixing-demixing one can be compensated by a larger entropy. We must point out that, on the basis of the investigation performed in Ref. [1] and the discussion made here below Eqs. (11)-(15), we do not expect this critical line to appear in the equilibrium phase diagram right above $\delta=0.46$. In fact, the interval $0.46<\delta<0.605$ corresponds to the hidden-binodal regime of Ref. [1], where the critical point at $x=1 / 2$ of the crescent-shaped line is metastable. Of course, knowledge of the behavior of the point at equimolar concentration alone is not sufficient to deliberate about the fate of the whole critical line. In principle, some portions of it might become stable for $\delta$ different from the value $\delta=0.605$ reported in [1]. However, this would imply a change in the topology of the critical lines with respect to that shown in Fig. 2 which we did not observe when fluctuations are taken into account (see Sec. IV), and we regard such an occurrence as rather unlikely. In summary, it is palusible that the value $\delta=0.605$ obtained in Ref. [1] sets the threshold for the appearance in the mean-field equilibrium phase diagram of the whole crescent-shaped critical line besides the point at $x=1 / 2$. The critical temperature along the crescentshaped line changes very little, typically by few percent, and it presents a shallow minimum at $x=1 / 2$. Along the other critical lines the qualitative behavior of the temperature is the same as in Fig. 1. For relatively low $\delta$, the minima of the critical temperature located along the critical line arising from the pure species are higher than the temperature at the ends 
of the crescent-shaped line, while the converse is true for $\delta$ larger than about 0.64 .

As $\delta$ is increased, the crescent grows towards larger and smaller concentrations, until for $\delta=0.65338$ the critical lines meet each other, resulting in the topology shown in Fig. 3 . When $\delta$ grows above this value, the former critical line originating from the pure components splits: the portions at low and high concentration, where the transition is mainly liquidvapor, join the crescent-shaped line so as to form a liquid-vapor critical line ranging from $x=0$ to $x=1$, while the part at intermediate concentration, where demixing prevails, remains connected to the demixing line at $x=1 / 2$ and detatches from the liquid-vapor line, giving a fork-shaped critical locus. The situation just described is illustrated in Fig. 团 for $\delta=0.7$. The liquid-vapor critical line has just one temperature minimum at $x=1 / 2$, in agreement with the above observation that for a liquid-vapor transition, increasing the concentration of the dilute component leads to a decrease of the critical temperature. The two temperature minima symmetric with with respect to $x=1 / 2$ are now located along the fork-shaped line. The relative temperature change along this line is however quite small, as already observed for the crescent-shaped line of Fig. 2. For $\delta$ just above the value 0.65338 that marks the boundary between the topology of Fig. 2 and that of Fig. 4 , the critical temperature at the tips of the fork-shaped line is higher than the minimum at $x=1 / 2$ on the liquid-vapor line, while the converse is true at higher $\delta$, including the value $\delta=0.7$ to which Fig. 4 refers. A similar behavior is found when comparing the minimum on the liquidvapor line with the temperature at the intersection of the fork with the mixing-demixing line.

If $\delta$ is further increased, the liquid-vapor line becomes more and more similar to a straight segment, as is to be expected since $\delta=1$ corresponds to a one-component fluid, whose critical density is obviously independent of the concentration. At the same time, the forkshaped line shrinks and moves to higher density, together with the mixing-demixing line. Strictly speaking, the fork disappears from the critical lines only in the one-component limit $\delta \rightarrow 1$. However, when $\delta$ gets larger than a value $\delta_{0}$ between 0.75 and 0.76 , this locus is certainly metastable, as the pressure along it is everywhere negative. We observe that this is a sufficient condition for metastability, but not a necessary one. In fact, according to Ref. [1], the portion of the fork near equimolar concentration has already disappeared from the equilibrium phase diagram for $\delta>0.708$. We are then left with two disconnected critical lines: the mixing-demixing one, that terminates at an endpoint, and the liquid-vapor one at lower density, as shown in Fig. 5 for $\delta=0.8$. For $\delta \rightarrow 1$, this topology evolves into that expected for the one-component fluid, as explained above: the mixing-demixing line eventually disappears, and the liquid-vapor line becomes a segment at constant density.

\section{HRT PHASE DIAGRAM}

As we said in the Introduction, HRT calculations were performed for an interaction that consists of a hard-sphere repulsive core and an attractive Yukawa tail, whose inverse-range parameter $z$ was set to $z=1.8$ for both like and unlike species. This value of $z$ is appropriate for representing the LJ potential [31] and it has been widely adopted in the literature. We illustrate our results by considering, for different values of the parameter $\delta$, several isothermal sections of the phase diagram, each of which corresponds to a single HRT run. In our opinion, this gives a clearer picture than the one that would be obtained by mapping the phase 
diagram at given $\delta$ on a single three-dimensional plot. In the following, the temperature will be identified with the corresponding reduced quantity $T^{*}=k_{\mathrm{B}} T / \epsilon$, and the asterisk will be omitted. Fig. 6 shows the phase diagram on the density-concentration plane at four different temperatures for $\delta=0.65$. Because of the symmetry of the model considered here, the phase diagram is obviously symmetric with respect to the equal-concentration axis $x=1 / 2$. We already pointed out that in the HRT the conditions of thermodynamic equilibrium that define the coexistence region are implemented by the theory itself. In fact, inside the domains shown in the figures the hessian determinant of the Helmholtz free energy is identically vanishing. We stress that this is essentially different from what is found in mean field-like approaches, where phase separation is marked by the appearance of spinodal surfaces in the $T$ - $\rho$ - $x$ space, which give spinodal lines upon intersecting with a plane at constant $T$ like those of the figure. Inside the regions bounded by these lines, the hessian of the free energy attains unphysically negative values. In the HRT, on the other hand, the hessian does not become negative, but it vanishes identically in a region of finite measure [32]. If we consider a curve at given temperature and pressure in the $\rho$ - $x$ plane, it is readily seen that along the portion of this curve that lies inside the domain where the hessian vanishes, the chemical potential of both species are identically constant. Therefore, such a domain is indeed the coexistence region of the mixture. This clearly appears from Fig. 7, which shows that the domains of Fig. 6 collapse into lines when they are plotted in the $P-\Delta \mu$ plane, where $P$ is the pressure, and $\Delta \mu=\mu_{1}-\mu_{2}$ is the difference between the chemical potentials of the two species. Because of the conditions of thermodynamic equilibrium, the coexistence regions in the $T-P-\Delta \mu$ space appear as "sheets" bounded by critical lines. Intersecting with a plane at constant $T$ then yields lines like those of Fig. 7 , terminating at critical points. Each point of these lines corresponds to an isobar of the domains of Fig. 6, i.e., to a tie-line. At a critical point, the tie-line reduces to a single point. We recall that in the $\rho-x$ plane a critical point is not in general an extremal point of the phase boundary [30], either in $\rho$ or in $x$, and it cannot be detected by just considering the shape of the $\rho-x$ coexistence boundaries. Critical points have been marked by dots in Fig. 6 and in the following figures that show the phase diagram in the $\rho$ - $x$ plane for different values of $\delta$.

The first panels of Figs. 6, 7 show the phase diagram at a temperature somewhat lower than the critical temperature $T_{c}^{0} \simeq 1.2$ of the pure components. Three distinct coexistence domains are present. The two at low density originate from the coexistence regions of the pure components, and they both terminate at a critical point. The high-density region at $\Delta \mu=0$, which is present also at temperatures above $T_{c}^{0}$, involves, at each fixed density, coexistence between two fluids at concentration $\bar{x}$ and $1-\bar{x}$ respectively, and terminates at a mixing-demixing critical point at $x=1 / 2$. Below a certain temperature $T_{t} \simeq 1.06$, however, the demixing region bifurcates into two branches (see the second panel of Fig. 7), each of which ends at a critical point with $\Delta \mu \neq 0, x \neq 1 / 2$. The former mixing-demixing critical point at $x=1 / 2$ has now become a first-order coexistence boundary, at which a mixed fluid at equal species concentration coexists with a demixed fluid at higher density similar to that found above $T_{t}$, consisting of two phases with concentrations symmetric with respect to $x=1 / 2$. At the temperature $T_{t}$ at which the bifurcation develops, the critical line at $x=1 / 2$ and the two critical lines generated by the symmetric branches for $T<T_{t}$ meet at a tricritical point. These critical lines can be visualized as the boundaries 
of the coexistence "sheets" in the $T-P-\Delta \mu$ space whose projections on the $P-\Delta \mu$ plane at constant $T$ are shown in Fig. 7. At the tricritical point, the mixed fluid at $x=1 / 2$ and the two phases that constitute the demixed fluid become critical simultaneously. Again, in the $\rho-x$ plane the tricritical point does not display any special feature that makes it immediately detectable. Inspection of the $\rho-x$ phase boundary alone does not allow one to tell whether the high-density coexistence region displays a single critical point at $x=1 / 2$, two symmetric critical points, or a tricritical point. By further lowering the temperature, the high- and low-density coexistence regions expand and get closer, until at a temperature $T_{d} \simeq 1.04$ each of the low-density regions meets the high-density one at a double critical point. The critical lines in the $\rho-x$ plane have the same topology as in Fig. 1. In particular, the tricritical point corresponds to the intersection of the mixing-demixing critical line at $x=1 / 2$ with the line that spans the concentration axis from $x=0$ to $x=1$. The latter actually results from projecting on the $\rho-x$ plane the two critical lines into which the mixingdemixing critical line bifurcates below $T_{t}$ and those originating from the critical points of the pure species. The two symmetric double critical points at $T=T_{d}$ where these critical lines meet in couples correspond to the temperature minima at $x \neq 1 / 2$ located on the critical line of Fig. 目. We observe that, unlike tricritical points, double critical points do not entail the intersection of topologically distinct critical lines. In fact, tricritical points are found in the model considered here because of its special symmetry, but they do not occur in real binary mixtures, while double critical points are frequently found in real mixtures, including mixtures of noble gases such as neon-krypton [33] and neon-xenon [34]. Below $T_{d}$, the coexistence domain consists of one connected region, without any critical point, as shown in the third and fourth panels of Fig. 7. A section of the phase diagram at $x=1 / 2$ in the $\rho-T$ plane is plotted in Fig. 8, showing the same behavior as in Fig. 2(d) of Ref. [1]. The HRT and the mean-field critical lines are compared in Fig. 9 for $\delta=0.5$. This value of $\delta$ is small enough to give the same topology of the critical lines in HRT and mean-field theory, save for an extremely short line at low density and nearly equimolar concentration obtained in mean field, which belongs to the metastable regime (see the discussion in the previous Section) and has not been reported here. The figure shows the projections of the critical lines both in the $\rho-x$ and in the $x-T$ planes; in the latter case, the mixing-demixing line at $x=1 / 2$ has not been shown. For the pure species, it is known from the comparison of the mean-field results with accurate simulation data for the critical constants of the Yukawa fluid with the same inverse range $z=1.8$ considered here [35], that mean-field theory underestimates the critical density by about $20 \%$ and overestimates the critical temperature by about $10 \%$. Basically the same differences are found by comparing the mean-field and the HRT results, as the HRT provides a very good determination of the critical point of LJ-like fluids [24,36],37]. Fig. 9 shows that similar discrepancies between mean field and HRT hold also for the critical loci of the binary system. We observe that the direction of the order parameter is little affected by the inclusion of fluctuations, at least for the present case where these do not change the topology of the critical lines.

According to our HRT calculations, the value $\delta=0.65$ is actually nearly coincident with the upper limit of $\delta$ for the topology that we have just described. The scenario at slightly larger $\delta$ is illustrated in Figs. 10, 11, which show the phase diagram in the $\rho-x$ and $P-\Delta \mu$ planes for $\delta=0.665$. At temperatures $T$ above 1.023, the phase diagram evolves as before: on lowering $T$, the high-density coexistence region bifurcates at a tricritical point, and each 
of the resulting branches merges with the low-density coexistence regions that originate from the pure species, so that just below $T=1.03$ there are no critical points left in the phase diagram. However, at $T=1.023$ two critical points reappear at low density. In the $P-\Delta \mu$ plane, this is marked by the appearance of two "twigs" that stick out of the lowdensity coexistence region, each terminating at a critical point, as shown in the third panel of Fig. 11. By slightly lowering $T$, these twigs grow longer, and for $T=T_{d}=1.022$ they meet at a critical double point located at $\Delta \mu=0$. At the same time, the low-density lobes of the coexistence region in the $\rho-x$ plane become very elongated, and coalesce at $x=1 / 2$. The topology of the critical lines is the same as in Fig. 2, where the crescent-shaped line at low density is that described by the new family of critical points. We remark that, according to mean-field theory, this line is extremely shallow with respect to the temperature. This is confirmed by the HRT results just reported, which give a relative variation of the critical temperature of about $0.1 \%$. Below $T_{d}$ (see the fourth panel of Fig. 10), the phase diagram presents an "island" of homogeneous, mixed fluid surrounded by a "sea" of phase-separated fluid. A section of the coexistence region at $x=1 / 2$ shows that in this regime we have two kinds of phase equilibria at equal species concentration: on the one hand, below the tricritical temperature $T_{t}$ a demixed fluid at high density coexists with a mixed one at intermediate density as before. On the other hand, this mixed fluid coexists, at slightly lower density, with another low-density mixed fluid. The latter phase equilibrium terminates for $T=T_{d}$ at a vapor-liquid critical point. Therefore, the phase diagram at $x=1 / 2$ exhibits both a critical and a tricritical point. On further lowering the temperature below $T_{d}$, the island of mixed fluid shown in Fig. 10 becomes smaller and smaller, until it is eventually swallowed by the coexistence region. Correspondingly, in the $\rho-T$ plane the two phase boundaries at $x=1 / 2$ meet at a triple point, where the two mixed fluids at different densities coexist with the demixed fluid [38]. Below the temperature of the triple point, the demixed fluid at high density coexists with the mixed one at low density. The phase diagram at equal species concentration is similar to Fig. 2(c) of Ref. [1] and to that shown below in Fig [12 for the case $\delta=0.67$. From the picture given above it is clear that the low-density lobes of the coexistence region meet at a temperature lower than that at which each lobe meets the high-density region. This in turn is lower than the temperature at which the tricritical point appears. Therefore, for the topology just discussed the liquid-vapor critical temperature $T_{d}$ is always lower than the tricritical temperature $T_{t}$.

A different situation arises as $\delta$ grows above 0.67. Figs. 13, 14 depict the evolution of the phase diagram for $\delta=0.68$. As before, a tricritical point is present at $x=1 / 2$, as shown by the bifurcation of the high-density coexistence region. On the other hand, on lowering the temperature the low-density branches of the coexistence region do not merge with the high-density one as before, but instead meet each other at a critical double point between $T=1.03$ and $T=1.025$. In this regime the isothermal sections of the phase diagram in the $\rho-x$ plane consist of two disconnected domains, with that at lower density spanning the whole density-concentration axis from $x=0$ to $x=1$. At about $T=1.025$, two twigs sprout out of the low-density coexistence domain in the $P-\Delta \mu$ plane. In such a situation this domain presents two symmetric critical points, corresponding to the ends of the twigs. On further lowering $T$, the twigs grow longer, and for $T$ between 1.015 and 1.01 each of them meets a branch of the high-density coexistence region at a critical double point. The low- and high-density coexistence domains then join at two points symmetric 
with respect to $x=1 / 2$, leaving between them an island of one-phase fluid (see Fig. 13), that eventually disappears at low temperature. This scenario is somehow the converse of that previously described for $\delta=0.665$, where the high-density coexistence domain merges with the low-density ones originating from the pure components, and the one-phase island inside the coexistence region is formed because of the twigs meeting each other at $x=1 / 2$.

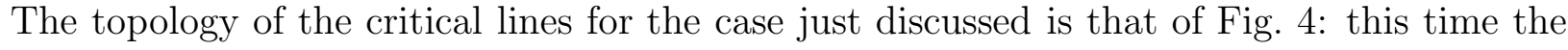
critical line that goes from $x=0$ to $x=1$ is not connected to the mixing-demixing critical line, and it has a temperature minimum at $x=1 / 2$, corresponding to the coalescence of the low-density coexistence regions into one connected domain. The mixing-demixing critical line intersects at the tricritical point two symmetric critical lines as before, which however do not meet those originating from the pure species. Instead, they merge with the lines described by the family of critical points that sprout out of the low-density coexistence region, resulting in the fork-shaped line of Fig. 国. This presents two symmetric temperature minima at the value of $T$ at which the critical lines meet each other. As already observed for the crescent-shaped line of Fig. 2 the temperature along the fork is actually almost constant, both according to mean-field theory and HRT, so that the minima are extremely shallow. The topology of the phase diagram at equimolar concentration is similar to the previous one illustrated in Fig. 12 for $\delta=0.67$. In particular, in a certain temperature interval including that where the two-phase region encloses a one-phase domain, there are two different phase equilibria at $x=1 / 2$. For the coupling parameter $\delta=0.68$ considered here, the liquid-vapor critical temperature $T_{d}$, which is the temperature minimum along the liquid-vapor critical line, is smaller than the tricritical temperature $T_{t}$, as for $\delta=0.665$. However, in the present topology where, on lowering $T$, the low-density coexistence domains merge with each other before they merge with the high-density one, nothing prevents the liquid-vapor critical point at $x=1 / 2$ from occurring at a higher temperature than the tricritical point. In the $P-\Delta \mu$ plane, this corresponds to the high-density coexistence region bifurcating at a temperature lower than that at which the low-density branches meet. Such a scenario in fact comes along starting from about $\delta=0.7$. The phase diagram at $x=1 / 2$ for this value of $\delta$ is shown in Fig. 15, and is the same at that of Fig. 2(b) of Ref. [1].

The phase behavior of the system for $\delta$ intermediate between the values 0.665 and 0.68 discussed above is worth being considered in more detail. Figs. 16, 17 show the phase diagram for $\delta=0.67$. On lowering the temperature, the low-density coexistence domains coalesce at about $T=1.0235$, and at a slightly lower temperature around $T=1.023$ the resulting region coalesce with the high-density coexistence domain. The phase diagram in the $\rho-x$ plane is then similar to that already shown for $\delta=0.68$, except that here the low- and high-density domains meet at nearly the same temperature. However, if one considers temperatures just above $T=1.0235$ it appears that, unlike what found for the $\delta$ studied above, the phase diagram displays six critical points, two for each of the disconnected domains that make up the coexisting region. This is clearly shown in the $P-\Delta \mu$ plane of Fig. 17 for $T=1.024$. In the present case it is not obvious to tell whether the topology of the critical lines in the $\rho-x$ plane is that of Fig. 2 or of Fig. [t, because it is difficult to ascertain which is the part of the coexistence region in the $P-\Delta \mu$ plane that is sprouting from the other, and consequently whether the high-density branches of the coexistence domain are bound to meet those originating from the pure species as for $\delta=0.655$, or the twigs as for $\delta=0.68$. We are therefore very close to the boundary between the two topologies of the critical loci, 
that corresponds to the scenario of Fig. 橉. This is marked by the presence of two tricritical points at non-equimolar concentration besides that at $x=1 / 2$. In such a situation the growth of a secondary structure (the twigs) from an already existing one (the main branch) is replaced by a bifurcation where both branches stem out of the tricritical point at the same temperature. For continuity reasons, we expect that in a very narrow range of $\delta$ values that includes 0.67 and is contained in the interval $0.665<\delta<0.68$, the configuration with six critical points shown in Fig. 17 can be found for the topology of the critical lines of both Fig. 2 and Fig. 目. If one considers the critical lines of Fig. 2, this occurs when the critical temperature at the ends of the low-density crescent-shaped line is higher than the minima located along the line originating from the pure species. In the case of Fig. 目, the requirement is that the local temperature minimum at $x=1 / 2$ along the liquid-vapor line must be lower not only than the tricritical temperature, but also than that of the critical points located at the tips of the fork-shaped line. It may also be worth pointing out that, strictly speaking, the watershed between the density-concentration phase diagrams of Fig. 10 and Fig. 13, that represent the two different ways by which a domain of one-phase fluid can be enclosed into the coexistence region, has not to coincide with the boundary between the critical lines just discussed, although the two are expected to occur for very similar values of $\delta$. In fact, according to mean-field theory, when the critical lines have the topology of Fig. [3, the coexistence region in the $\rho-x$ plane still looks like that of Fig. 10. This implies that there is an extremly narrow range of $\delta$ where the topology of the critical lines is as in Fig. 因, and the topology of the phase diagram in the $\rho-x$ plane is as in Fig. 10. In the $P-\Delta \mu$ plane such a regime is marked by the twigs joining the bifurcation of the high-density coexistence region at a temperature higher than that at which the low-density branches meet each other. We have not checked whether this scenario comes along also in the HRT, or instead it is replaced by the converse one, where the critical lines have the topology of Fig. 2, and the phase diagram in the $\rho-x$ plane has the topology of Fig. 13.

Further information on the nature of the phase equilibria is obtained by considering the tie-lines, i.e., the lines in the $\rho-x$ plane that connect the phases at coexistence at a certain temperature and pressure. A few tie-lines of the mixture with $\delta=0.67$ are shown in Fig. 18 for $T=1.024$ (left panel) and $T=1.023$ (right panel), corresponding respectively to the second and fourth panel of Fig. 16. We have chosen to report the tie-lines for the case $\delta=0.67$ because of its particularly rich phase diagram, several features of which are separately found also for different values of $\delta$. In both panels of Fig. 18, the high-density portion of the coexistence region is characterized by the presence of a demixed fluid which, as said above, consists of two phases at the same density and concentrations symmetric with respect to the equal-concentration axis. As a consequence, the average density coincides with that of the coexisting phases irrespective of their relative amount in the demixed fluid, resulting in strictly vertical tie-lines. Since both panels refer to temperatures below the tricritical temperature $T_{t}$, the phase equilibrium just described does not extend down to the left boundary of the high-density coexistence domain. At a certain density $\rho_{\mathrm{D}}$, the two coexisting fluids at symmetric concentrations $\bar{x}, 1-\bar{x}$ coexist in turn with a fluid at a lower density $\rho_{\mathrm{M}}$ and equimolar concentration. The densities $\rho_{\mathrm{M}}, \rho_{\mathrm{D}}$ are the boundaries of the first-order coexistence domain that separates the mixed-fluid and demixed-fluid regions of Fig. 12 for $T<T_{t}$. In the $\rho-x$ plane, this three-phase equilibrium takes place in a trianglelike domain bounded by three tie-lines meeting in couples at the points $\left(\rho_{\mathrm{D}}, \bar{x}\right),\left(\rho_{\mathrm{D}}, 1-\bar{x}\right)$, 
$\left(\rho_{\mathrm{M}}, 1 / 2\right)$. These lie very close to (but do not exactly coincide with) the two oblique lines and the leftmost vertical line shown in the high-density region of Fig. 18. The two symmetric portions of the high-density region of the left panel that lie above and below the three-phase domain correspond in the $P-\Delta \mu$ plane to the branches of the bifurcation that stems from the line at $\Delta \mu=0$ (see Fig. 17). Each of them presents coexistence between two phases which differ both in density and concentration, terminating at a critical point where the tie-line reduces to a single point. In the right panel the critical points are absent, as the high- and low-density coexistence regions have merged into one connected domain. The left panel also shows the tie-lines shrinking at the four critical points located on the two symmetric lobes of the low-density coexistence region. An interesting feature that appears from the arrangement of the tie-lines in this region is the presence of two more domains of three-phase coexistence, such that the coexisting phases have concentrations that lie on the same side of the $x=1 / 2$ axis. At temperatures at which the coexistence region is connected and encloses an island of homogeneous fluid as in the right panel, this sort of three-phase equilibrium could be expected just on the basis of the fact that different tie-lines cannot intersect each other. However, the presence in the low-density region of the two critical points associated with the twigs in the $P-\Delta \mu$ plane implies that three-phase coexistence is observed in this region even at temperatures at which it has not yet merged with the highdensity coexistence domain. We also observe that in the neighborhood of the $x=1 / 2$ axis where the two lobes of the low-density region coalesce, the tie-lines are nearly horizontal, meaning that the concentration of the coexisting phases are very similar. At exactly $x=1 / 2$ azeotropy occurs, i.e., one finds a purely liquid-vapor transition between two phases at equal concentration, as already observed above in connection with the phase diagram at $x=1 / 2$ in the $\rho$ - $T$ plane. The critical point topping the liquid-vapor coexistence curve at equimolar concentration in Figs. 12, 15 is therefore an azeotropic critical point.

As $\delta$ increases above 0.7 , the curvature of both the low- and high-density coexistence boundaries in the $\rho-x$ plane rapidly decreases, and the pressure at which the bifurcation of the high-density coexistence region takes place gets closer and closer to that of the coexisting vapor and liquid phases on the low-density region at $x=1 / 2$. As a consequence, the one-phase island bounded by the coexistence region becomes smaller and involves a fluid at nearly equimolar concentration. One can then ask whether this feature of the phase diagram will disappear at a certain $\delta_{0}<1$, or instead only in the limit $\delta \rightarrow 1$. The former case corresponds to a situation where for $\delta>\delta_{0}$ the high-density coexistence region does not undergo any bifurcation in the $P-\Delta \mu$ plane, but it always presents a mixing-demixing critical point at $x=1 / 2, \Delta \mu=0$. At low enough temperature, this critical point meets the low-density coexistence region at a first-order phase boundary. The topology of the critical lines in the $\rho-x$ plane is that of Fig. 5: the mixing-demixing line terminates at a critical enpoint where the low- and high-density coexistence regions meet. The phase diagram at $x=1 / 2$ corresponding to this scenario is that of Fig. 2(a) of Ref. [1]. As before, the equimolar mixture displays both a liquid-vapor transition that occurs below a certain critical temperature and involves two mixed fluids of different densities, and a mixing-demixing transition at high density. In this case, however, the transition between the mixed liquid and the demixed fluid is always second-order down to the temperature of the endpoint, below which the demixed fluid coexists with a mixed vapor of much lower density. The situation where the contact between the low- and the high-density coexistence 
region always occurs at two distinct points symmetric with respect to $x=1 / 2$ until $\delta$ gets equal to 1 is instead consistent with the phase diagram remaining qualitatively similar to that found for $\delta=0.7$ with critical lines as in Fig. \&, albeit the fork-shaped line will become vanishingly small as $\delta$ approaches 1 and the mixing-demixing line will be pushed to higher density. The phase diagram of the equimolar mixture in the $\rho$ - $T$ plane will qualitatively look as that of Fig. 15. As a consequence, as long as one has $\delta<1$ there will be a small temperature interval between the triple point and the tricritical point, where the transition between the mixed and the demixed liquid is first order. This is the scenario advocated in Ref. [39] on the basis of modified hypernetted chain (MHNC) calculations on a symmetric LJ mixture, while the simulations performed in Ref. [1] on a square-well system supported the existence of a critical endpoint at high enough $\delta$. Some calculations that we previously performed on a hard-sphere LJ mixture using a $100 \times 100$ density-concentration grid also suggested that for $\delta=0.8$ the phase diagram of the system presents a critical endpoint. However, the present results for a hard-core Yukawa potential shown in Figs. 19, 20 indicate that for $\delta=0.8$, the mixture most likely does not have a critical endpoint: in fact, in a certain narrow temperature range the coexistence region in the $\rho$ - $x$ plane still encloses a domain of one-phase fluid with concentration varying in a small interval around $x=1 / 2$. Since the inverse range $z=1.8$ of the Yukawa potential considered here gives a fair representation of the LJ interaction [31, the difference between this behavior and that reported in Ref. [19] most probably does not depend on some intrinsic difference between the two interactions, but just on the fact that the higher resolution entailed by the $150 \times 150$ grid used in this work allows one to study the contact between the low- and high-density coexistence regions with better accuracy than in Ref. [19], thereby uncovering the presence of a small one-phase domain. We should observe that the case $\delta=0.8$ actually lies at the limit of resolution even for the larger grid employed here: the homogeneous domain inside the coexistence region in the $\rho-x$ plane extends only one gridpoint in the $\rho$-direction, while the phase diagram in the $P-\Delta \mu$ plane does not present any of the features associated with the occurrence of such a domain on the scale of the figure, so that it cannot be clearly distinguished from the kind of diagram one would expect in the presence of a critical endpoint. In particular, there is no sign of the bifurcation of the high-density coexistence region in the $P-\Delta \mu$ plane that we expect if the low- and high-density regions have to meet at two points of nonequimolar concentration according to the scenario of Fig. 14. This most probably depends on the fact that, for the relatively high value of $\delta$ considered here, the densities of the mixed and demixed liquids at coexistence are so close, that their difference is comparable to the mesh size. As a consequence, the differences in the pressures and chemical potentials of neighboring but non-coexisting points will also become comparable to the numerical errors. A little numerical noise in the $P-\Delta \mu$ plane due to the finite mesh used in the calculation is in fact always present, as shown by close inspection of the relevant figures, but only at high $\delta$ does this become a hindrance for a clear description of the phase behavior. We did not pursue any investigation for $\delta>0.8$, because a fortiori in such a regime the resolution allowed by the grid used here would not enable us to discriminate between the occurrence of a critical endpoint, or an extremely small region of homogeneous fluid that undergoes a first-order demixing transition on slightly increasing the density. Therefore, we are not in a position to say whether a critical endpoint at $x=1 / 2$ will eventually appear near $\delta=1$, or instead a very weak first-order transition will survive up to $\delta=1$. Another issue that 
is difficult to elucidate within the resolution of the present calculation concerns the fate of the twigs discussed before in connection with the phase diagram topology of Figs. 13, 14. As noted above, even at temperatures at which the low-density coexistence regions that originate from the pure species have coalesced into one connected domain, the latter can still show two symmetric critical points. When this is the case, the low-density coexistence region in the $P-\Delta \mu$ plane presents two small twigs, each of them ending at a critical point. These twigs could either disappear at high enough $\delta$, or persist for $\delta$ arbitrarily close to 1. If a critical endpoint at $x=1 / 2$ never appears and the topology of the critical lines in the $\rho-x$ plane is that of Fig. Tuntil $\delta$ gets equal to unity, the latter case corresponds to a situation where the low- and high-density coexistence regions will always meet at two double critical points. As discussed above, mean-field theory indeed gives two double critical points located along the fork-shaped line, but for $\delta$ close to 1 they are certainly metastable since they correspond to negative pressure. If instead the twigs disappear before $\delta$ reaches 1 , there will be a value of $\delta$ above which the critical points of the high-density coexistence region meet the low-density region at two points of first-order coexistence. As a consequence, the contact takes place at two critical endpoints with concentrations symmetric with respect to $x=1 / 2$ rather than at two double critical points. This is the situation considered in Ref. [39]. In such a case the critical lines in the $\rho-x$ plane have still the same topology as in Fig. 因, except that the temperature minima along the fork-shaped line have now to coincide with the tips of the fork, which correspond to the two endpoints. It can also be worthwhile observing that in principle the twigs are not incompatible with a critical endpoint at equimolar concentration. In fact, this could even give rise to a scenario where the presence of the endpoint is consistent with the high- and low-density coexistence regions meeting at non-equimolar concentration. Specifically, if the critical points located at the ends of the twigs meet the high-density coexistence region at two symmetric critical endpoints with $x \neq 1 / 2$, nothing prevents the point at equimolar concentration of this region from remaining critical, until it also meets the low-density region at a critical endpoint. We remark that such a possibility hinges on the presence of the twigs and the related critical points on the low-density coexistence region. In the converse situation where the critical endpoints at $x \neq 1 / 2$ result from the contact between two points of first-order coexistence on the low-density domain and two critical points on the high-density one, the requirement that the point at $x=1 / 2$ be critical is untenable, as pointed out in Ref. [39]. The scenario just depicted is somewhat suggestive, as it could account for both the presence of a critical endpoint at $x=1 / 2$ above a certain value of $\delta$ reported in simulation studies [ []], and the failure to observe the low- and high-density coexistence regions coalescing at $x=1 / 2$ found in the present investigation as well as in [39]. However, we must point out that at the present stage we do not have any solid evidence that this possibility does actually occur, so that we must regard it as a purely speculative conjecture.

\section{CONCLUSIONS}

We have used the HRT to perform an investigation of the phase diagram of symmetric binary mixtures as a function of the unlike-to-like interaction ratio $\delta$. The microscopic interaction adopted consisted of a hard-core repulsion plus an attractive Yukawa tail potential

with inverse range $z=1.8$. Such a potential has been used many times in liquid-state 
theory to describe a simple LJ-like fluid. For each value of $\delta$ considered, results for the coexistence regions were obtained on the whole density-concentration plane at several temperatures. The resulting phase portrait was related both to the different topologies of the mean-field critical lines that come along as $\delta$ is varied in the interval $0<\delta<1$, and to the behavior predicted by mean-field theory and simulation results [i] for a certain special class of the systems considered here, namely equimolar mixtures at molar fraction $x=1 / 2$. A feature of the HRT that is particularly useful is that the domains of coexisting phases are straightforwardly obtained by the theory as the loci where the conditions of thermodynamic equilibrium between different phases are satisfied, without any need of enforcing them $a$ posteriori. Fulfillment of these conditions is shown by the collapse of the isothermal sections of the coexistence regions on lines of the $P-\Delta \mu$ plane, $P$ being the pressure, and $\Delta \mu$ the difference between the chemical potentials of the two species. This property makes also easy to identify the critical points exhibited by the phase diagram at a certain temperature.

According to the results previously obtained by mean-field theory and simulations, the phase diagram of symmetric mixtures at equimolar concentration is characterized by three different regimes, depending on the value of $\delta$ : at low $\delta(\delta<0.605$ according to meanfield theory [1]) a mixing-demixing critical line joins a mixing-demixing coexistence curve at a critical point, thereby generating a tricritical point. For $\delta$ closer to $1(\delta>0.708$ in mean field [1]), the mixing-demixing critical line joins a liquid-vapor coexistence curve at a critical endpoint. Finally, in a narrow interval in $\delta$ intermediate between the above regimes, the mixture shows both a liquid-vapor coexistence curve and a mixing-demixing one at higher densities, topped respectively by a critical and a tricritical point. Our investigation clearly shows the tricritical point regime as well as the intermediate one. The latter is predicted to occur starting from about $\delta>0.65$, in agreement with the simulation results for a square-well mixture [ [], but it lingers on for larger values of $\delta$ than those given by simulation: according to simulation, the endpoint regime is reached for $\delta>0.68$, while according to HRT $\delta=0.7$ is still in the "transition" or intermediate regime, as shown in Fig. 15. As a matter of fact, we did not find any clear evidence of the endpoint regime, since our calculations indicate that the mixture is likely to be in the intermediate regime for $\delta$ as high as 0.8 . However, on increasing $\delta$, the transition region quickly moves to high density, and the finite resolution allowed by the $150 \times 150$ grid used here becomes insufficient to fully uncover the topology of the phase diagram in this parameter range. For this reason we did not investigate the behavior of the model for $\delta>0.8$. The results obtained in this work are in qualitative agreement with a study based on the MHNC integral equation for a LJ mixture [39], according to which no critical endpoint at equimolar concentration is present up to at least $\delta=0.81$. Before drawing any definite conclusion about the resilience of the intermediate topology in the theoretical HRT and MHNC results compared with the simulations and the mean-field approximation, the role of the specific interaction adopted should be elucidated. In fact, the HRT and MHNC calculations were performed on a HCY and on a LJ potential respectively, while in the simulations a hard-sphere plus square-well was used. As observed in Sec. [, the HCY potential was also employed in other investigations based on the MSA [21], the ORPA [22], and the SCOZA [23] theories, all of which yield for the phase diagram the same qualitative picture found in mean-field theory. However, we do not see any general reason why the independency of the interaction profile which is intrinsic to the mean-field phase behavior should always hold also for more sophisticated approaches, 
specifically for the HRT. Moreover, one can also imagine a scenario where the existence of a critical endpoint at equimolar concentration is not prevented by the behavior observed here for the phase diagram in the $\rho-x$ plane, namely the fact that, even for relatively high $\delta$, the low- and high-density coexistence regions meet first at two points symmetric with respect to $x=1 / 2$ rather than at $x=1 / 2$. However, the present limits in resolution of our numerical calculation do not allow us to say whether this conjecture is actually relevant for the system studied.

An interesting issue that was considered here is how the phase portraits corresponding to the topologies mentioned above look like, if one moves off the plane of equimolar concentration. In particular, the intermediate regime is marked by the presence, in a certain temperature interval, of a phase coexistence domain with a hole of homogeneous fluid inside it. This comes along according to two distinct scenarios that can occur as the temperature is lowered: for $0.65<\delta<0.67$, the two low-density coexistence regions originating from the pure species meet the high-density coexistence region associated with the mixing-demixing transition, and subsequentely they meet each other, leaving a domain of mixed fluid enclosed inside the two-phase region. For $\delta>0.67$, first the two low-density coexistence regions meet each other, and then the resulting connected domain meets the high-density coexistence region at two points symmetric with respect to concentration $x=1 / 2$, leaving some one-phase fluid in between. In the former case, the temperature $T_{c}$ of the liquid-vapor critical point at $x=1 / 2$ is always lower than the tricritical temperature $T_{t}$, while in the latter case $T_{c}$ can be either lower or higher than $T_{t}$, depending on $\delta$. The present calculation gives $T_{t}<T_{c}$ starting from about $\delta=0.7$.

Because of the existence of both a liquid-vapor and a demixing transition, the critical loci show both a line that spans the concentration axis and connects the critical points of the pure components, and a mixing-demixing line at $x=1 / 2$. The tricritical point topology corresponds to a situation where these lines are connected, and the character of the transition changes continuously from liquid-vapor at $x=0$ or $x=1$ to mixing-demixing at $x=1 / 2$. In the endpoint regime, which as said above was not observed in the present investigation, the critical lines are instead disconnected, and the transition along the line that connects the critical points of the pure species is essentially of liquid-vapor type. The intermediate regime between these topologies is characterized by the presence of a further critical line at concentrations ranging in a certain interval centered at $x=1 / 2$, which was referred to above as either the "crescent" or the "fork" line. The number of critical points that are found for a certain isothermal section of the phase diagram depends on the relative location of the temperature extrema along the critical lines. The behavior found by the present HRT calculation agrees qualitatively with that given by mean-field theory. In particular, the system is predicted to have up to six critical points at a certain temperature. According to HRT, this occurs for a very narrow range of $\delta$ values contained in the interval $0.665<\delta<0.68$. At the boundary between the "crescent" and the "fork" topologies of the critical lines, the system has two tricritical points at symmetric non-equimolar concentrations besides that at concentration $x=1 / 2$. In HRT, this particular topology occurs for a value of $\delta$ close to 0.67 , to be compared with the mean-field result $\delta=0.65338$. In both cases, these values lie very near the boundary between the two different types of one-phase "holes" in the phase coexistence region described above. When $\delta$ is such that mean-field theory and HRT predict the same qualitative topology of the phase diagram, the quantitative discrepancy 
between the critical loci given by the two approaches is similar to that found for the pure species.

This investigation shows that symmetric mixtures, despite their conceptual simplicity, exhibit a very rich phase behavior. Like real mixtures, these systems have both liquidvapor and mixing-demixing transitions. However, while in real mixtures the liquid-vapor and the mixing-demixing regimes generally correspond to states that differ widely in density and pressure, in symmetric mixtures these transitions can instead be located in the same region of the thermodynamic space. As a consequence, they tend to compete with each other, so that even a small variation in the relative strength of the interactions expressed by the parameter $\delta$ is sufficient to bring about significant qualitative changes in the phase diagram. The results presented here are also relevant for Ising ferrofluids in the presence of a magnetic field. More generally, they show that HRT is capable of providing a comprehensive description and resolving even subtle features of the phase behavior of the model. This ability could prove useful to systematically study beyond the mean-field level also the phase diagram of more realistic, non-symmetric model mixtures that depend one more than just one parameter. 


\section{REFERENCES}

[1] N. B. Wilding, F. Schmid, and P. Nielaba, Phys. Rev. E 58, 2201 (1998).

[2] H. Zhang and M. Widom, Phys. Rev. E 49, R3591 (1994).

[3] B. Groh and S. Dietrich, Phys. Rev. E 50, 3814 (1994).

[4] P. C. Hemmer and D. Imbro, Phys. Rev. A 16, 380 (1977).

[5] J. M. Tavares, M. M. Telo da Gama, P. I. C. Teixeira, J. J. Weis, and M. J. P. Nijmeijer, Phys. Rev. E 52, 1915 (1995).

[6] A. Oukouiss and M. Baus, Phys. Rev. E 55, 7242 (1997).

[7] M. A. Zaluska-Kotur and L. A. Turski, Phys. Rev. A 41, 3066 (1990).

[8] M. J. P. Nijmeijer, A. Parola, and L. Reatto, Phys. Rev. E 57, 465 (1998).

[9] F. Schinagl, H. Iro, and R. Folk, Eur. Phys. J. B 8, 113 (1999).

[10] R. O. Sokolovskii, Phys. Rev. B 61, 36 (2000).

[11] A. Z. Panagiotopoulos, N. Quirke, M. Stapleton, and D. J. Tildesley, Mol. Phys. 63, 527 (1988).

[12] J. R. Recht and A. Z. Panagiotopulos, Mol. Phys. 80, 843 (1993).

[13] D. G. Green, G. Jackson, E. de Miguel, and L. F. Rull, J. Chem. Phys. 101, 3190 (1994).

[14] E. de Miguel, E. M. del Río, and M. M. Telo da Gama, J. Chem. Phys. 103, 6188 (1995).

[15] N. B. Wilding, Phys. Rev. E 55, 6624 (1997).

[16] C. Caccamo, D. Costa, and G. Pellicane, J. Chem. Phys. 109, 4498 (1998).

[17] A. Parola and L. Reatto, Phys. Rev. A 44, 6600 (1991).

[18] D. Pini, L. Reatto, and A. Parola, J. Phys. Cond. Matt. 9, 1417 (1997).

[19] D. Pini, A. Parola, and L. Reatto, J. Stat. Phys. 100, 13 (2000).

[20] A. Parola, D. Pini, M. Tau, and L. Reatto, to appear in J. Phys. Cond. Matt.

[21] G. Kahl, E. Schöll-Paschinger, and A. Lang, Monatshefte für Chemie 132, 1413 (2001).

[22] E. Schöll-Paschinger, D. Levesque, J.-J. Weis, and G. Kahl, Phys. Rev. E 64, 011502 (2001).

[23] E. Schöll-Paschinger, Ph.D. thesis, Technische Universität Wien, 2002.

[24] A. Parola and L. Reatto, Adv. Phys. 44, 211 (1995).

[25] G. A. Mansoori, N. F. Carnahan, K. E. Starling, and T. W. Leland, J. Chem. Phys. 54, 1523 (1971).

[26] E. W. Grundke and D. Henderson, Mol. Phys. 24, 269 (1972).

[27] L. L. Lee and D. Levesque, Mol. Phys. 26, 1351 (1973).

[28] W. F. Ames, Numerical Methods for Partial Differential Equations, Academic Press, New York, 1977.

[29] P. H. van Konynenberg and R. L. Scott, Phil. Trans. R. Soc. London Ser. A 298, 495 (1980).

[30] J. S. Rowlinson and F. L. Swinton, Liquids and Liquid mixtures, Butterworths, London, 1982.

[31] Henderson, D., Waisman, E., Lebowitz, J. L., and Blum, L., 1978, Mol. Phys. 35, 241.

[32] A. Parola, D. Pini, and L. Reatto, Phys. Rev. E 48, 3321 (1993).

[33] N. J. Trappeniers and J. A. Schouten, Physica 73, 546 (1974).

[34] A. Deerenberg, J. A. Schouten, and N. J. Trappeniers, Physica 101A, 459 (1980).

[35] D. Pini, G. Stell, and N. B. Wilding, Mol. Phys. 95, 483 (1998).

[36] M. Tau, A. Parola, D. Pini, and L. Reatto, Phys. Rev. E 52, 2644 (1995).

[37] C. Caccamo, G. Pellicane, D. Costa, D. Pini, and G. Stell, Phys. Rev. E 60, 5533 (1999). 
[38] Strictly speaking, at the triple point there are four coexisting phases, since the demixed fluid consists of two homogeneous fluids at concentrations symmetric with respect to $x=1 / 2$.

[39] O. Antonevych, F. Forstmann, and E. Diaz-Herrera, Phys. Rev. E 65, 061504 (2002). 


\section{FIGURES}

FIG. 1. Density-concentration projection of the mean-field critical lines of a symmetric mixture with interaction parameter $\delta=\epsilon_{12} / \epsilon_{11}=0.4$. The total density $\rho$ is the sum of the densities $\rho_{1}, \rho_{2}$ of the components, and the concentration $x$ is defined as $\rho_{1} / \rho$. The open dots mark the locations of the local minima in the critical temperature. The arrows indicate the direction of the order parameter (see text).

FIG. 2. Same as Fig. 1 for $\delta=0.65$.

FIG. 3. Same as Fig. 1 for $\delta=0.65338$.

FIG. 4. Same as Fig. 1 1 for $\delta=0.7$.

FIG. 5. Same as Fig. 1 for $\delta=0.8$.

FIG. 6. Isothermal sections of the coexistence region of a symmetric HCY mixture in the $\rho-x$ plane according to the HRT. The inverse interaction range is equal to $z=1.8$ and the interaction ratio is equal to $\delta=0.65$. The dots mark the locations of the critical points.

FIG. 7. Same as Fig. 6 in the pressure-chemical potential plane. $\Delta \mu=\mu_{1}-\mu_{2}$ is the difference between the chemical potentials of the components. Note how the points of the coexistence regions shown in Fig. 6 collapse into lines as a consequence of the conditions of thermodynamic equlibrium.

FIG. 8. Phase diagram in the $\rho$-T plane of the HCY mixture with $\delta=0.65$ for the special case of equimolar concentration $x=1 / 2$. Open and full dots denote respectively the $\lambda$-line and the first-order phase boundary.

FIG. 9. Critical lines in the $\rho$ - $x$ plane (upper panel) and $x$-T plane (lower panel) of the HCY mixture with $\delta=0.5$. Dotted lines: mean-field theory. Full dots: HRT. In the upper panel, the solid line is a guide for the eye obtained by smoothly interpolating between the dots, and the arrows indicate the direction of the order parameter. The order parameter along the mixing-demixing critical line at $x=1 / 2$ is not shown here for clarity and is parallel to the $x$-axis according to both mean field and HRT. In the lower panel, the mixing-demixing critical line is not shown.

FIG. 10. Same as Fig. 6 for $\delta=0.665$.

FIG. 11. Same as Fig. 7 for $\delta=0.665$. 
FIG. 12. Same as Fig. 8 for $\delta=0.67$. The letters V, L denote respectively the vapor and mixed liquid phase.

FIG. 13. Same as Fig. 6 for $\delta=0.68$.

FIG. 14. Same as Fig. 7 for $\delta=0.68$.

FIG. 15. Same as Fig. 12 for $\delta=0.7$.

FIG. 16. Same as Fig. 6 for $\delta=0.67$.

FIG. 17. Same as Fig. 7 for $\delta=0.67$.

FIG. 18. Coexistence region in the $\rho-x$ plane for $\delta=0.67$ at $T=1.024$ (left panel) and $T=1.023$ (right panel), corresponding to the second and fourth panels of Fig. 16, showing a few tie-lines connecting phases at coexistence. The different shades of gray give a measure of pressure (black: low pressure; white: high pressure).

FIG. 19. Same as Fig. 6 for $\delta=0.8$. The critical points in the second panel are not shown, because for this value of $\delta$ the resolution of our numerical calculation does not allow us to locate them.

FIG. 20. Same as Fig. 团 for $\delta=0.8$. In the first panel, the high-density coexistence region at $\Delta \mu=0$ does not appear as its pressures are outside the scale of the figure. 
This figure "fig1.png" is available in "png" format from: http://arxiv.org/ps/cond-mat/0211485v1 
This figure "fig2.png" is available in "png" format from: http://arxiv.org/ps/cond-mat/0211485v1 
This figure "fig3.png" is available in "png" format from: http://arxiv.org/ps/cond-mat/0211485v1 
This figure "fig4.png" is available in "png" format from: http://arxiv.org/ps/cond-mat/0211485v1 
This figure "fig5.png" is available in "png" format from: http://arxiv.org/ps/cond-mat/0211485v1 
This figure "fig6.png" is available in "png" format from: http://arxiv.org/ps/cond-mat/0211485v1 
This figure "fig7.png" is available in "png" format from: http://arxiv.org/ps/cond-mat/0211485v1 
This figure "fig8.png" is available in "png" format from: http://arxiv.org/ps/cond-mat/0211485v1 
This figure "fig9.png" is available in "png" format from: http://arxiv.org/ps/cond-mat/0211485v1 
This figure "fig10.png" is available in "png" format from: http://arxiv.org/ps/cond-mat/0211485v1 
This figure "fig11.png" is available in "png" format from: http://arxiv.org/ps/cond-mat/0211485v1 
This figure "fig12.png" is available in "png" format from: http://arxiv.org/ps/cond-mat/0211485v1 
This figure "fig13.png" is available in "png" format from: http://arxiv.org/ps/cond-mat/0211485v1 
This figure "fig14.png" is available in "png" format from: http://arxiv.org/ps/cond-mat/0211485v1 
This figure "fig15.png" is available in "png" format from: http://arxiv.org/ps/cond-mat/0211485v1 
This figure "fig16.png" is available in "png" format from: http://arxiv.org/ps/cond-mat/0211485v1 
This figure "fig17.png" is available in "png" format from: http://arxiv.org/ps/cond-mat/0211485v1 
This figure "fig18.png" is available in "png" format from: http://arxiv.org/ps/cond-mat/0211485v1 
This figure "fig19.png" is available in "png" format from: http://arxiv.org/ps/cond-mat/0211485v1 
This figure "fig20.png" is available in "png" format from: http://arxiv.org/ps/cond-mat/0211485v1 